\title{
Composição, riqueza e heterogeneidade da flora arbórea da bacia do rio São João, RJ, Brasil
}

\author{
Fabrício Alvim Carvalho ${ }^{1,2,4}$, Marcelo Trindade Nascimento² e Ary Teixeira Oliveira Filho ${ }^{3}$
}

\author{
Recebido em 23/04/2007. Aceito em 26/12/2007
}

\begin{abstract}
RESUMO - (Composição, riqueza e heterogeneidade da flora arbórea da bacia do rio São João, RJ, Brasil). Estudos florísticos vêm apontando a região da bacia do rio São João, no Estado do Rio de Janeiro, como detentora de uma riqueza e diversidade de espécies arbóreas particularmente altas. Entretanto, tais afirmativas são baseadas em estudos locais, não existindo ainda uma análise de sua flora arbórea em nível regional. A partir da compilação de inventários florísticos e fitossociológicos de 20 trechos de floresta ombrófila de baixada (30-300 m), o presente trabalho aborda a composição, riqueza e heterogeneidade da flora arbórea da bacia do rio São João. A compilação incluiu apenas as árvores com DAP $\geq 2,5 \mathrm{~cm}$, que foram identificadas em nível específico. A amostra da flora arbórea totalizou 460 espécies, 231 gêneros e 62 famílias. Estes números reafirmam que a região é realmente detentora de uma flora arbórea com alta diversidade, inclusive com diversas espécies raras ou vulneráveis à extinção, podendo ser considerada como uma das mais ricas entre as de Floresta Atlântica do Sudeste brasileiro. Análises multivariadas detectaram uma alta heterogeneidade florística entre os fragmentos florestais, contribuindo na elevada riqueza regional encontrada. Os padrões de similaridade sugerem que a principal fonte desta heterogeneidade provém dos diferentes estados de conservação das áreas.
\end{abstract}

Palavras-chave: análise multivariada, conservação, Floresta Atlântica ombrófila de baixada, flora regional

ABSTRACT - (Composition, richness and heterogeneity of the tree flora in the São João river basin, Rio de Janeiro State, Brazil). Floristic studies have indicated the São João river basin, in the state of Rio de Janeiro, SE Brazil, as containing particularly high tree species richness and diversity. Nevertheless, such statements are based on local studies only since an analysis of the tree flora at a regional level has yet to be produced. This study compiles floristic and phytosociological surveys of 20 patches of low altitude rain forest (30-300 m of elevation) in the São João river basin to produce an analysis of the composition, diversity and heterogeneity of the tree flora. The compilation included only trees with $\mathrm{dbh} \geq 2.5 \mathrm{~cm}$ that were identified to the species level. The tree-flora sample totaled 460 species, 231 genera and 62 families. These figures confirm that the region actually shelters a tree flora with high species diversity, including many considered rare or vulnerable to extinction, and may be considered one of the richest among the Atlantic Forests of SE Brazil. Multivariate analysis detected high floristic heterogeneity among forest remnants, which contributes to this high regional richness. Patterns of floristic similarity suggest that the main source of this heterogeneity is the difference in conservation status of each patch.

Key words: multivariate analysis, conservation, lowland Atlantic rain forest, regional flora

\section{Introdução}

A Floresta Atlântica do Estado do Rio de Janeiro que, no passado, cobria a quase totalidade do seu território, encontra-se hoje restrita a apenas $19 \%$ de sua cobertura original, e suas maiores manchas florestais são observadas nas vertentes das cadeias montanhosas da Serra do Mar, onde a altitude geralmente ultrapassa a cota de 500 m (Fundação SOS Mata Atlântica 2002). A região da planície litorânea do centro-norte fluminense, originalmente coberta em sua maior parte por florestas de baixas altitudes $(<250 \mathrm{~m})$, foi alvo durante séculos de intensas perturbações antropogênicas, intensificadas nas últimas sete décadas com o expressivo crescimento da população urbana e rural, ocasionado aumento na extração madeireira e a substituição de suas florestas por áreas agrícolas (Dean 1996). A paisagem atual dessa região encontra-se representada por fragmentos florestais de variados tamanhos, mas em sua grande maioria pequenos, isolados, perturbados e imersos em extensas matrizes antrópicas, como pastos e monoculturas (Fundação SOS Mata Atlântica 2002; Kierulff et al. 2005).

Localizada na planície litorânea do centro-norte do Estado do Rio de Janeiro, a bacia do rio São João estende-

\footnotetext{
${ }^{1}$ Universidade de Brasília, Departamento de Ecologia, Programa de Pós-Graduação em Ecologia, C. Postal 04457, 70919-970 Brasília, DF, Brasil

2 Universidade Estadual do Norte Fluminense, Laboratório de Ciências Ambientais, Av. Alberto Lamego 2000, Parque Califórnia, 28015-620 Campos dos Goytacazes, RJ, Brasil

3 Universidade Federal de Lavras, Departamento de Ciências Florestais, 37200-000 Lavras, MG, Brasil

${ }^{4}$ Autor para correspondência: fabricioalvim@yahoo.com.br
} 
se por cerca de $3.000 \mathrm{~km}^{2}$ e ainda mantém vários remanescentes de Floresta Atlântica de baixada (Fundação SOS Mata Atlântica 2002; Primo \& Volker 2003; Kierulff et al. 2005). Estes remanescentes estão totalmente inseridos no assim chamado Corredor Ecológico da Serra do Mar (ou Corredor Sul da Mata Atlântica), área de extrema importância ecológica e conservacionista, por deter o que outrora constituiu a maior extensão contínua de Floresta Atlântica, entre os Estados do Rio de Janeiro e Paraná (Ayres et al. 2005). Conforme proposto no plano de estudos estratégicos desse Corredor, informações florísticas sobre as regiões que o compõem são necessárias para subsidiar a implementação de ações conservacionistas (Ayres et al. 2005).

Informações sobre a flora da bacia são recentes e, somente nas duas últimas décadas, vêm sendo realizados estudos florísticos e fitossociológicos, através dos trabalhos de Scarano et al. (1997), Scarano et al. (1998), Borém \& Ramos (2001), Borém \& Oliveira Filho (2002), Carvalho et al. (2006a;b), Guedes-Bruni et al. (2006a;b), Pessoa \& Oliveira (2006), Scarano (2006) e Carvalho et al. (2007). Estes estudos vêm apontando os remanescentes florestais da bacia do rio São João como detentores de elevada riqueza arbórea. Entretanto, as informações geradas limitam-se a análises em escalas locais (Carvalho et al. 2004; Carvalho et al. 2006a), sendo poucos os estudos que procuraram detectar padrões florísticos em escalas mais amplas, em nível regional.

Assim, este estudo visa preencher essa lacuna do conhecimento, agregando informações sobre a flora arbórea dos remanescentes de Floresta Atlântica de baixada da bacia do rio São João, por meio da compilação de inventários florísticos e fitossociológicos realizados na região e da aplicação de análises multivariadas, com o objetivo de produzir a primeira análise regional da composição, riqueza e heterogeneidade florística nessa bacia. Parte-se da hipótese de que os fragmentos florestais remanescentes são ricos em espécies e, em função da alta heterogeneidade de habitats, possuem baixa similaridade florística entre si, contribuindo para a ocorrência de uma elevada riqueza em nível regional.

\section{Material e métodos}

Área de estudo - A bacia do rio São João (coordenada central $22^{\circ} 25^{\prime} \mathrm{S} ; 42^{\circ} 15^{\prime} \mathrm{O}$ ) está localizada na planície litorânea da região centro-norte do Estado do Rio de Janeiro, e possui uma área de cerca de $3.000 \mathrm{~km}^{2}$, abrangendo oito municípios: Cachoeiras de Macacú, Rio Bonito, Silva Jardim, Casimiro de Abreu, Cabo Frio, São Pedro d'Aldeia, Armação de Búzios e Araruama (Fig. 1). Ações antrópicas, como a retificação dos leitos do rio
São João, a construção de oleodutos, gasodutos, linhas de transmissão de energia, ferrovias e rodovias, aliados ao desmatamento ocorrido nas décadas passadas, são os principais causadores atuais da degradação florestal na região (Primo \& Volker 2003; Kierulff et al. 2005). Devido à grande pressão antrópica na região, em 2002 foi criada a Área de Proteção Ambiental (APA) do rio São João/Mico-Leão-Dourado, abrangendo a quase totalidade da bacia, com a finalidade de proteger e conservar os mananciais, regular o uso dos recursos hídricos e o parcelamento do solo, garantindo o uso racional dos recursos naturais e protegendo os remanescentes de Floresta Atlântica e o patrimônio ambiental e cultural da região (Primo \& Volker 2003). No domínio da APA estão incluídas duas Reservas Biológicas Federais, Poço das Antas e União, além de mais de uma dezena de RPPNs (Reserva Particular do Patrimônio Natural) que ali foram implementadas (Carvalho et al. 2004; Kierulff et al. 2005).

A bacia do rio São João possui topografia predominantemente de planície costeira, sendo formada por serras $(21 \%)$, planaltos $(13 \%)$, colinas ou morrotes mamelonares (32\%), áreas de baixadas aluviais sujeitas ao alagamento permanente ou periódico do solo (30\%) e restingas (4\%) (Primo \& Volker 2003). Predomina na região o clima tropical úmido com estação chuvosa no verão e sem inverno pronunciado. As temperaturas médias anuais são elevadas durante quase todo o ano, sendo o clima enquadrado pela classificação de Koeppen (1948) no tipo As (tropical chuvoso com estação seca no inverno) (Primo \& Volker 2003). A quantidade de chuva oscila entre 1.500 e $2.000 \mathrm{~mm}$, sendo os meses de junho a agosto os menos chuvosos e de menores temperaturas, e os meses de novembro a março os mais chuvosos e de maiores temperaturas (Primo \& Völker 2003). No intervalo de 1993-2002, período em que foi conduzida a maior parte dos levantamentos florísticos deste estudo, a temperatura média anual foi de $25,5^{\circ} \mathrm{C}$, sendo julho o mês de temperatura média mais baixa $\left(21,8{ }^{\circ} \mathrm{C}\right)$, e os meses de janeiro e fevereiro os de temperaturas médias mais altas $\left(29,0{ }^{\circ} \mathrm{C}\right.$ ) (Pessoa \& Oliveira 2006). Em relação à precipitação, foi observado, no período, um valor médio anual de $1.995 \mathrm{~mm}$, sendo o mês de dezembro o de maior valor $(320 \mathrm{~mm})$ e julho o de menor valor (38,9 mm) (Pessoa \& Oliveira 2006). Os solos encontrados nessa região são classificados como Latossolo Vermelho-Amarelo, nas florestas de baixa altitude não alagáveis, Cambissolo, nas florestas de elevadas altitudes, e solos Gleissolos, Organossolos e Neossolos Flúvicos, nas florestas das planícies de inundação e aluviais (Primo \& Volker 2003).

As florestas de baixada (Floresta Ombrófila Densa de Terras Baixas e Submontana, de 0 a 250 m, sensu 


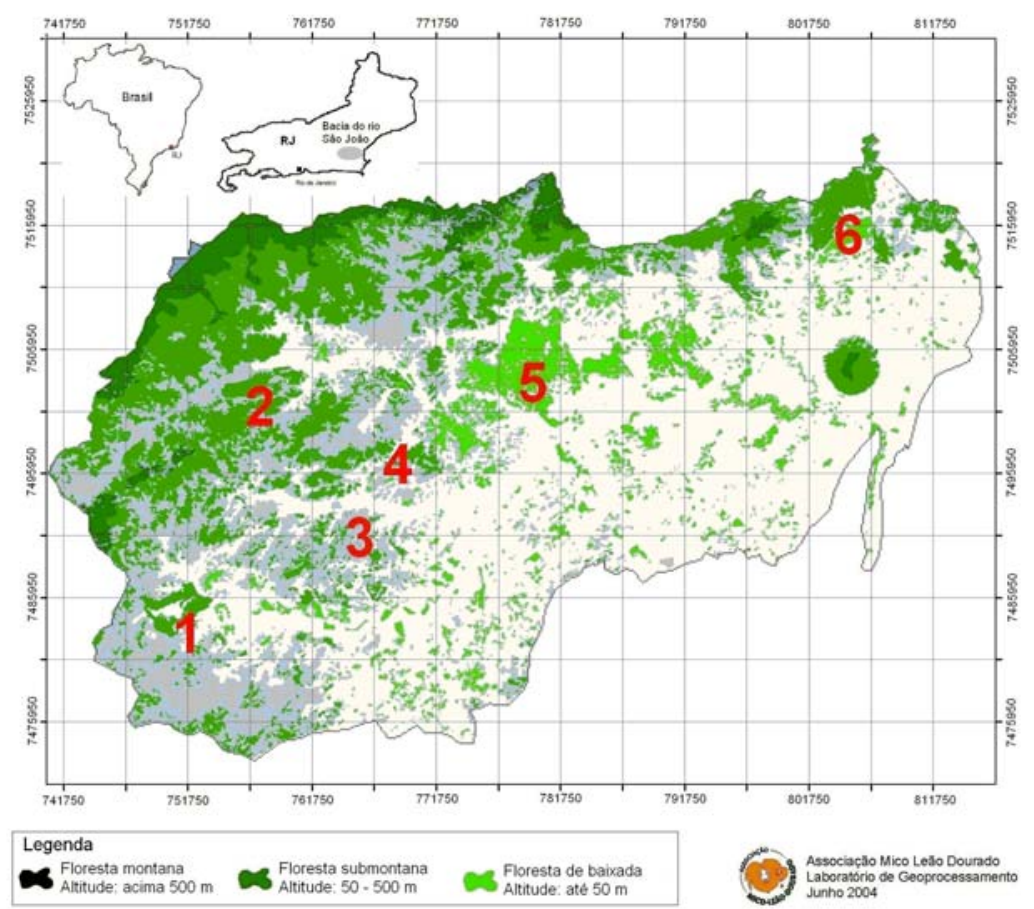

Figura 1. Localização geográfica e vegetação florestal remanescente da bacia do rio São João, RJ, Brasil, e das seis localidades de Floresta Atlântica ombrófila de baixas altitudes, cujos fragmentos florestais foram analisados quanto à composição e diversidade da flora arbórea. Localidades: (1) Fazenda Rio Vermelho, (2) Fazenda Biovert, (3) Região de Imbaú, (4) Região de Boqueirão, (5) Reserva Biológica de Poço das Antas, (6) Reserva Biológica União.

Veloso et al. 1991) ocupavam originalmente cerca de $66 \%$ da extensão da bacia. O restante da vegetação era composto por florestas inundáveis (Floresta Ombrófila Densa Aluvial, sensu Veloso et al. 1991), com cerca de $30 \%$, e restingas (Formação pioneira com influência marinha), com aproximadamente $4 \%$ da área da bacia. Atualmente, a cobertura vegetal dessa região é constituída principalmente por campos antrópicos (pastos, monoculturas, áreas urbanas), áreas de regeneração florestal (capoeiras) e remanescentes de florestas de baixada, sujeitas ou não ao alagamento (Primo \& Volker 2003). Apesar de fragmentada, a vegetação da bacia do rio São João destaca-se por ainda manter vários remanescentes de Floresta Atlântica e por abrigar espécies da fauna ameaçadas de extinção, tais como o mico-leãodourado (Leontopithecus rosalia) e a preguiça-de-coleira (Bradypus torquatus), entre outras (Kierulff et al. 2005). Das espécies que compõem sua flora, muitas se encontram com populações reduzidas, devido ao extrativismo secular na região, como o guanandi (Symphonia globulifera) e o jequitibá-branco (Cariniana legalis), dentre outras (IBDF 1981).

Análise dos dados - Com base na bibliografia disponível, foi realizada uma compilação das espécies arbóreas inventariadas em estudos fitossociológicos de fragmentos de Floresta Atlântica ombrófila de baixas altitudes na bacia do rio São João. Para homogeneizar a amostra em termos de forma de crescimento arbóreo, foi adotado $2,5 \mathrm{~cm}$ como o limite mínimo de inclusão de DAP (diâmetro a altura do peito a $1,30 \mathrm{~m}$ acima do solo). A circunscrição das fisionomias amostradas em "Floresta Atlântica ombrófila de baixas altitudes" refere-se às florestas situadas em altitude entre 30 e $300 \mathrm{~m}$, sem alagamento permanente ou estacional do solo, conforme adotado por Oliveira Filho \& Fontes (2000). Este termo aproxima-se da fusão das florestas enquadradas nas fisionomias Floresta Ombrófila Densa de Terras Baixas (0-50 m) e Submontana (50-500 m), segundo o sistema de classificação da vegetação do IBGE (Veloso et al. 1991), resguardada a diferença nas faixas altitudinais.

Foram analisados dados oriundos de estudos realizados por diferentes autores em 20 fragmentos florestais, distribuídos por seis localidades distintas na bacia do rio São João (Fig. 1). As localidades foram: (1) Fazenda Rio Vermelho (um fragmento florestal analisado, total de 0,4 ha de área amostral); (2) Fazenda Biovert (dois fragmentos florestais analisados, total de 0,72 ha de área amostral); (3) Região de Imbaú (cinco fragmentos florestais analisados, total de 1,0 ha de área amostral); (4) região de Boqueirão (dois fragmentos florestais analisados, total de 0,4 ha de área amostral); 
(5) Reserva Biológica de Poço das Antas (sete fragmentos florestais analisados, total de 3,9 ha de área amostral); e (6) Reserva Biológica União (três fragmentos florestais analisados, total de 1,2 ha de área amostral). Informações sobre os fragmentos florestais analisados estão sumarizadas na Tab. 1.

Para a realização das análises, foi preparado um banco de dados binários (presença/ausência), contendo a relação das espécies arbóreas inventariadas nas localidades acima referidas. Na composição deste banco de dados, foram considerados somente os indivíduos identificados em nível de espécie. Julgou-se seguro o uso do nível específico, visto que todas as identificações foram realizadas no herbário do Jardim Botânico do Rio de Janeiro (RB), utilizando-se as mesmas coleções de referências e auxílio dos mesmos especialistas. Todos os inventários utilizaram o sistema de classificação em famílias, de Cronquist (1981). Para reduzir o problema com sinonímias, as espécies tiveram seus binômios confirmados e atualizados por meio do "software" do índice de espécies do Royal Botanical Garden of Kew (Royal Botanical Garden 1993), ou por meio de literatura especializada.

Para a análise da composição florística, foram compiladas todas as espécies presentes nos inventários consultados, tratadas em nível de espécie, gênero e família. Estes dados também foram utilizados para a análise da riqueza, assim como também foi feita uma projeção do número total de espécies a partir dos estimadores não paramétricos 'jackknife' de primeira e segunda ordem, capazes de projetar a riqueza total de espécies a partir da riqueza de espécies das amostras e da heterogeneidade entre elas (Heltsche \& Forrester 1983). Para a análise da heterogeneidade florística, o banco de dados binários foi utilizado em dois métodos multivariados: (1) uma análise de agrupamento com

Tabela 1. Informações sobre os 20 fragmentos de Floresta Atlântica ombrófila de baixas altitudes da bacia do rio São João, RJ, Brasil, analisados quanto à composição da flora arbórea regional. DAP: limite de inclusão de diâmetro (cm); Área: área amostral (ha); Alt.: altitude (m); S: número de espécies (Se: número de espécies identificadas em nível específico e utilizadas nas análises).

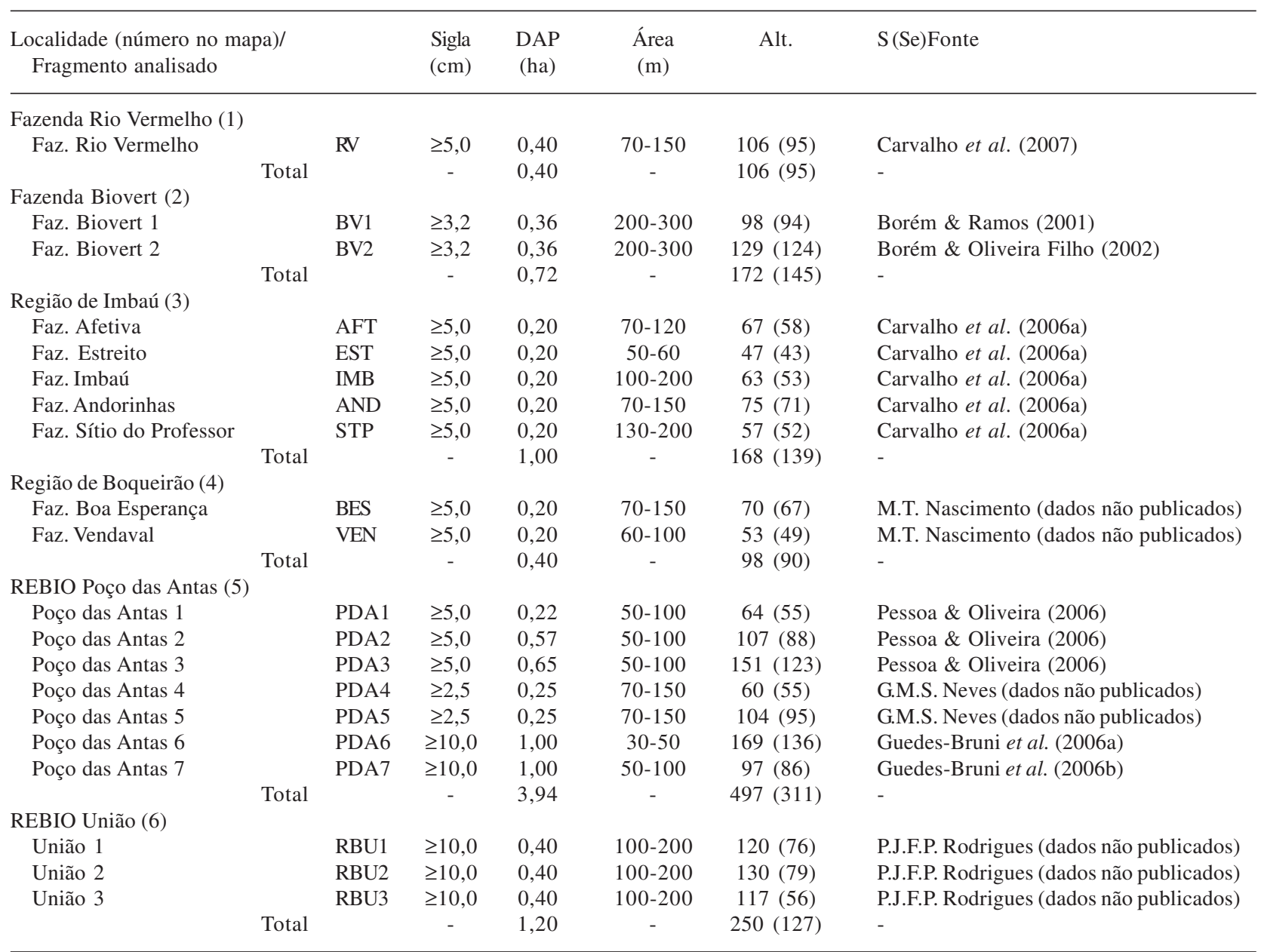

REBIO: Reserva Biológica - Unidade de Conservação Federal; Faz. Fazenda - propriedade particular. 
emprego do índice de Jaccard como medida de similaridade florística, e o método de agrupamento das médias não ponderadas (UPGMA) para representação gráfica sob a forma de dendrograma (Kent \& Coker 1992); (2) e uma análise de coordenadas principais a partir do método de distâncias médias ('average distance'), para ordenação espacial dos dados e representação sob a forma de gráfico 'biplot' (Kent \& Coker 1992). As análises multivariadas foram realizadas utilizando-se o programa MVSP versão $3.13 \mathrm{~m}$ (Kovach 2004).

Como forma de auxiliar no subsídio a estratégias de conservação, as espécies de maior freqüência ( $\geq 50 \%)$ nos remanescentes foram classificadas em grupos ecológicos, segundo suas características sucessionais e síndrome de dispersão. Esta classificação foi baseada em diversos autores que se utilizaram destes dados nos seus estudos, abrangendo trabalhos em florestas do domínio da Floresta Atlântica. As espécies foram classificadas segundo suas características sucessionais em pioneiras ( $\mathrm{Pi})$, secundárias iniciais ( $\mathrm{Si}$ ) e secundárias tardias (St), segundo proposto por Gandolfi et al. (1995). Para as síndromes de dispersão, adotou-se a terminologia de Van der Pijl (1982), sendo as espécies classificadas em zoocóricas, anemocóricas e autocóricas.

\section{Resultados e discussão}

A compilação de todas as espécies arbóreas presentes nos 20 fragmentos florestais das seis localidades (total de 7,66 ha de área amostral) de Floresta Atlântica ombrófila de baixas altitudes da bacia do rio São João totalizou 460 espécies, distribuídas em 231 gêneros e 62 famílias. As famílias com maior riqueza de espécies foram Leguminosae (sensu lato) (66 espécies), Lauraceae (45), Myrtaceae (34), Euphorbiaceae (24), Moraceae (20), Annonaceae (19), Rubiaceae (18) e Sapotaceae (16). Estas oito famílias apresentaram grande contribuição para a diversidade arbórea regional, concentrando $52,6 \%$ do total de espécies. Dentre os gêneros, os mais ricos em espécies foram Ocotea (22) e Eugenia (16), seguidos de Myrcia (8), Inga (8), Guatteria (7), Mollinedia (7), Trichilia (7) e Miconia (7), que, juntos, concentraram $17,8 \%$ do total de espécies.

O padrão de riqueza de famílias/gêneros encontrado corrobora com o descrito por Oliveira Filho \& Fontes (2000) para o domínio da Floresta Atlântica ombrófila de baixa altitude do Sudeste brasileiro, com acentuada riqueza de espécies arbóreas das famílias Leguminosae, Myrtaceae e Lauraceae, e dos gêneros Eugenia e Ocotea (Tab. 2). Vale ressaltar que a riqueza de espécies da família Myrtaceae, tida como a mais rica nas análises de Oliveira Filho \& Fontes (2000), está subestimada na presente análise, devido ao grande número de táxons identificados em nível de gênero ou morfoespécie, e que, portanto, não atenderam aos critérios de inclusão utilizado nesta compilação de dados. Provavelmente, a inclusão destas espécies após correta identificação elevaria a família Myrtaceae ao nível de maior riqueza de espécies na bacia do rio São João.

Outros estudos realizados em trechos de Floresta Atlântica ombrófila de baixas altitudes no Estado do Rio de Janeiro também apresentaram as famílias Leguminosae, Myrtaceae e Lauraceae como as detentoras de maior riqueza de espécies (Kurtz \& Araújo 2000; Moreno et al. 2003; Peixoto et al. 2004; R.R. Guedes-Bruni, dados não publicados). Dentre as principais diferenças com relação aos trechos de maior altitude (florestas ombrófilas de elevadas altitudes, acima de $500 \mathrm{~m}$ ), destaca-se a elevada riqueza de Sapotaceae, Meliaceae e Bignoniaceae nas florestas de terras baixas, sendo estas pouco expressivas nas florestas de maior altitude, onde Melastomataceae, Asteraceae, Solanaceae e Apocynaceae assumem maior riqueza (Lima \& GuedesBruni 1997; Oliveira Filho et al. 2005; R.R. GuedesBruni, dados não publicados). H.C. Lima (dados não publicados) sugere que, embora a família Leguminosae apresente elevada riqueza de espécies ao longo da Floresta Atlântica do Estado, esta riqueza tende a aumentar excepcionalmente nas florestas de baixas altitudes. Tais padrões florísticos concordam com os descritos por Oliveira Filho \& Fontes (2000) para o domínio da Floresta Atlântica do Sudeste brasileiro (Tab. 2) e, numa escala mais ampla, aos padrões encontrados por Gentry (1995) para as florestas tropicais do norte da América do Sul e América Central.

As riquezas totais de espécies projetadas pelos estimadores 'jackknife' foram de 599 (primeira ordem) e 647 (segunda ordem), sugerindo elevada riqueza regional de espécies arbóreas. Uma comparação dos valores de riquezas totais de espécies projetadas pelos estimadores 'jackknife' com dados da literatura permite afirmar com segurança que a flora arbórea dos remanescentes florestais da bacia do rio São João é particularmente rica. Em sua análise da riqueza da flora arbórea da Floresta Atlântica ombrófila de baixas altitudes do Sudeste brasileiro, Oliveira Filho \& Fontes (2000) listaram 1.475 espécies arbóreas em 27 trechos florestais (Tab. 2). Isto significa dizer que a riqueza de espécies registrada na bacia do rio São João representa $31 \%$ do total encontrado por estes autores para o Sudeste brasileiro e, considerando os valores projetados pelos estimadores 'jackknife', esta riqueza se aproxima dos $45 \%$.

Ressalta-se ainda que muitas espécies presentes nos inventários utilizados na compilação de dados da bacia 
934 Carvalho, Nascimento \& Oliveira Filho: Composição, riqueza e heterogeneidade da flora arbórea da bacia do rio...

Tabela 2. Famílias e gêneros de maior riqueza de espécies arbóreas na composição regional da Floresta Atlântica ombrófila de baixas altitudes da bacia do rio São João, RJ, Brasil, e comparação com o padrão descrito por Oliveira Filho \& Fontes (2000) para o domínio da Floresta Atlântica do Sudeste brasileiro. S: número de espécies; \%: percentagem da riqueza total; n: número de fragmentos florestais.

\begin{tabular}{|c|c|c|c|c|c|c|c|c|}
\hline \multirow{2}{*}{$\begin{array}{l}\text { Bacia do Rio São } \\
\text { Baixas altitudes } \\
(\mathrm{n}=20) \\
\text { Família }\end{array}$} & \multicolumn{2}{|c|}{$S=460$} & \multirow{2}{*}{$\begin{array}{l}\text { Sudeste brasileiro } \\
\text { Baixas altitudes } \\
(\mathrm{n}=27)\end{array}$} & \multicolumn{2}{|c|}{$S=1475$} & \multirow{2}{*}{$\begin{array}{l}\begin{array}{l}\text { Sudeste brasileiro } \\
\text { Elevadas altitudes } \\
(\mathrm{n}=21)\end{array} \\
\text { Família }\end{array}$} & \multicolumn{2}{|c|}{$\mathrm{S}=1280$} \\
\hline & $\mathrm{S}$ & $\%$ & & S & $\%$ & & S & $\%$ \\
\hline Leguminosae & 66 & 14,3 & Myrtaceae & 203 & 13,8 & Myrtaceae & 203 & 15,9 \\
\hline Lauraceae & 45 & 9,8 & Leguminosae & 198 & 13,4 & Leguminosae & 142 & 11,1 \\
\hline Myrtaceae & 34 & 7,4 & Rubiaceae & 84 & 5,7 & Melastomataceae & 82 & 6,4 \\
\hline Euphorbiaceae & 24 & 5,2 & Lauraceae & 78 & 5,3 & Lauraceae & 81 & 6,3 \\
\hline Moraceae & 20 & 4,3 & Melastomataceae & 76 & 5,2 & Rubiaceae & 76 & 5,9 \\
\hline Annonaceae & 19 & 4,1 & Sapotaceae & 49 & 3,3 & Euphorbiaceae & 35 & 2,7 \\
\hline Rubiaceae & 18 & 3,9 & Chrysobalanaceae & 43 & 2,9 & Monimiaceae & 35 & 2,7 \\
\hline Sapotaceae & 16 & 3,5 & Euphorbiaceae & 42 & 2,8 & Solanaceae & 31 & 2,4 \\
\hline Gênero & $S$ & $\%$ & Gênero & $S$ & $\%$ & Gênero & S & $\%$ \\
\hline Ocotea & 20 & 4,3 & Eugenia & 70 & 4,7 & Eugenia & 73 & 5,7 \\
\hline Eugenia & 18 & 3,9 & Myrcia & 37 & 2,5 & Miconia & 48 & 3,8 \\
\hline Myrcia & 8 & 1,7 & Ocotea & 36 & 2,4 & Ocotea & 40 & 3,1 \\
\hline Inga & 8 & 1,7 & Miconia & 35 & 2,4 & Myrcia & 32 & 2,5 \\
\hline Guatteria & 7 & 1,5 & Pouteria & 36 & 2,4 & Mollinedia & 27 & 2,1 \\
\hline Miconia & 7 & 1,5 & Marlierea & 22 & 1,5 & Inga & 24 & 1,9 \\
\hline Mollinedia & 7 & 1,5 & Erythroxylum & 21 & 1,4 & Solanum & 21 & 1,6 \\
\hline Trichilia & 7 & 1,5 & Inga & 20 & 1,4 & Gomidesia & 17 & 1,3 \\
\hline
\end{tabular}

do rio São João não foram incluídas por não estarem identificadas em nível específico, especialmente aquelas pertencentes às famílias Myrtaceae, Lauraceae e Sapotaceae. A adição dessas espécies certamente aumentaria de forma considerável a listagem, ressaltando a relevância da bacia do rio São João no contexto fitogeográfico da Floresta Atlântica do Sudeste, e especialmente no cenário da conservação da flora desta região, que, segundo Thomaz et al. (1998), destaca-se pelo elevado nível de endemismo de espécies de plantas, especialmente as pertencentes à família Myrtaceae.

A análise das similaridades florísticas entre os fragmentos evidenciou uma elevada heterogeneidade regional em composição de espécies arbóreas (Fig. 2). De acordo com Kent \& Coker (1992), duas áreas podem ser consideradas floristicamente similares quando o valor do índice de Jaccard ultrapassa 0,5. Os níveis de similaridade entre as localidades foram todos inferiores a este limite e, portanto, baixos. Somente o maior deles $(0,46)$ se aproximou do limite. De fato, do total de 460 espécies, 147 (32\%) foram restritas a apenas uma localidade, e 99 (22\%) a duas localidades. Este resultado concorda com a hipótese inicial, uma vez que o índice de similaridade de espécies pode ser utilizado para expressar a diversidade regional (diversidade beta), no sentido em que, quanto menor for a similaridade entre as áreas, maior será a diversidade regional, devido a uma maior diferenciação florística em função da alta heterogeneidade entre as amostras (Felfili \& Felfili 2001; Felfili et al. 2004).

Segundo Gentry (1988) a riqueza e a distribuição de espécies lenhosas nas florestas tropicais está relacionada a cinco gradientes principais: o intercontinental, o latitudinal, o de precipitação, o altitudinal e o edáfico. Considerando que, embora de difícil controle, algumas destas condições (latitudinal,

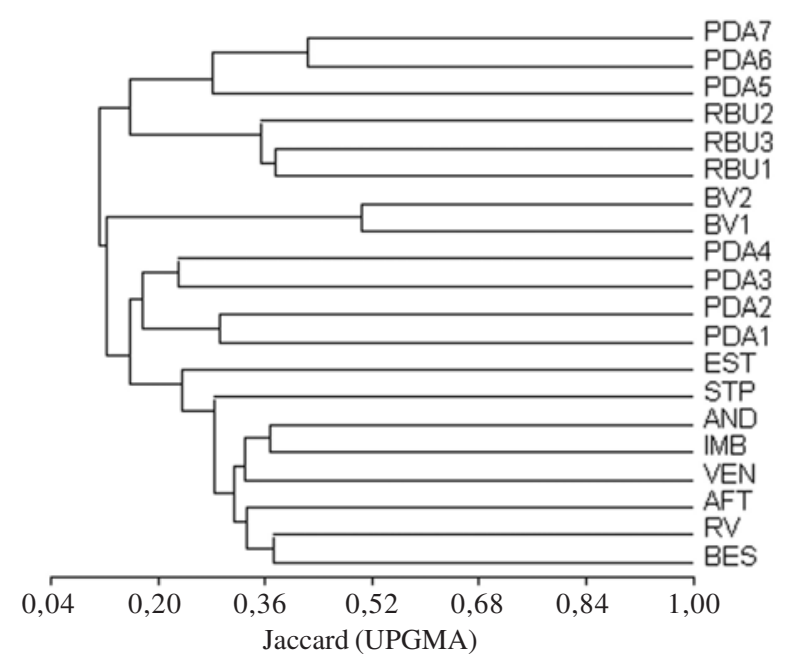

Figura 2. Dendrograma de similaridade (índice de Jaccard) produzido por análise de agrupamento (método de ligação UPGMA) da composição de espécies arbóreas entre os 20 fragmentos de Floresta Atlântica ombrófila de baixas altitudes na bacia do rio São João, RJ, Brasil. Siglas dos fragmentos conforme Tab. 1. 
precipitação e altitudinal) se assemelham entre os fragmentos analisados, a explicação mais plausível para a elevada dissimilaridade obtida parece estar relacionada a outros dois fatores: a proximidade geográfica entre os fragmentos (gradiente intercontinental) e ao histórico de perturbação antropogênica, conforme vêm sendo sugerido em estudos recentes da Floresta Atlântica (Carvalho et al. 2006a; Pereira et al. 2006; Carvalho et al. 2007).

Os fragmentos das localidades de Boqueirão (BES e VEN), Fazenda Rio Vermelho (RV) e Região de Imbaú (AFT, AND, EST, IMB e STP), dentre os mais similares entre si, são aqueles mais próximos geograficamente e também aqueles em pior estado de conservação, pois sofreram mais impactos antrópicos devido à localização em propriedades privadas sem a efetiva proteção (Carvalho et al. 2004; Carvalho et al. 2006a; Carvalho et al. 2007). Portanto, estas localidades tendem a compartilhar espécies arbóreas com estratégias de dispersão e estabelecimento mais eficientes mediante distúrbios (pioneiras e secundárias iniciais) (Budowisk 1965; Hubbell et al. 1999). Tais espécies também tendem a ter distribuição eco-geográfica mais ampla e, considerando a proximidade entre os fragmentos, a serem compartilhadas entre um maior número de áreas (Hubbell et al. 1999; Tabarelli \& Peres 2002). Já os fragmentos das localidades Reserva Biológica de Poço das Antas (PDA1 a PDA7) e Reserva Biológica União (RU1 a RU3) são precisamente os mais preservados da bacia do rio São João. A similaridade entre elas, ainda que bem mais baixa que entre o primeiro grupo (Fig. 2), é muito provavelmente proporcionada pela presença de espécies características de estádios sucessionais mais tardios (secundárias tardias), ausentes ou raras nas localidades mais perturbadas, visto que uma distância maior os separa (Fig. 1). A localidade Fazenda Biovert (fragmentos BV1 e BV2), embora também apresente estado de conservação inferior aos supracitados (Borém \& Ramos 2001; Borém \& Oliveira Filho 2002), distingue-se das demais (Fig. 2) por estar localizada mais próxima à base da cadeia montanhosa da Serra do Mar e, muito provavelmente, por sofrer maior influência da flora de elevadas altitudes adjacente, além de estar mais distante geograficamente dos demais (Fig. 1). Esta diferenciação florística, provavelmente mais ligada à altitude, corrobora o que é descrito para a Floresta Atlântica em escalas geográficas mais amplas, onde o papel mais forte é relacionado ao regime de chuvas e à altitude, com suas correspondentes variações de temperatura (Torres et al. 1997; Oliveira Filho \& Fontes 2000; Scudeller et al. 2001).

A análise de coordenadas principais (Fig. 3) praticamente mostrou os mesmos padrões da análise de agrupamentos (Fig. 2), porém, evidenciou melhor a formação dos grupos florísticos em relação aos fatores de distribuição das espécies supracitados. O eixo 1 representa o estado de conservação dos fragmentos, deixando os fragmentos mais preservados (PDA e RU) à direita do gráfico; já o eixo 2 representa a proximidade entre os fragmentos, separando os fragmentos BV, mais próximos da cadeia montanhosa da serra do mar, dos demais. A explicação destes dois eixos atingiu apenas $27,5 \%$, sugerindo que outros fatores contribuem para a elevada heterogeneidade encontrada. De fato, conforme citado por Gentry (1988), muitos são os fatores responsáveis pela elevada heterogeneidade florística em escalas local e regional em florestas tropicais, heterogeneidade esta reconhecida como um dos seus padrões mais característicos da Floresta Atlântica brasileira (Mori et al. 1981; Thomaz et al. 1997; Tabarelli \& Mantovani 1999; Oliveira Filho \& Fontes 2000). Deve-se considerar ainda que fatores como condições edáficas e microclimáticas não foram incluídos nas análises, e que parte das variações encontradas pode estar relacionada também ao uso de diferentes metodologias entre os trabalhos utilizados (Tab. 1). Estes fatores de difícil controle podem dificultar a interpretação dos resultados (Torres et al. 1997; Oliveira Filho \& Fontes 2000; Scudeller et al. 2001).

Uma outra abordagem que vem sendo aplicada em estudos fitogeográficos consiste no uso de espécies indicadoras de fitofisionomias, como forma de auxiliar na interpretação dos dados florísticos e na distinção das

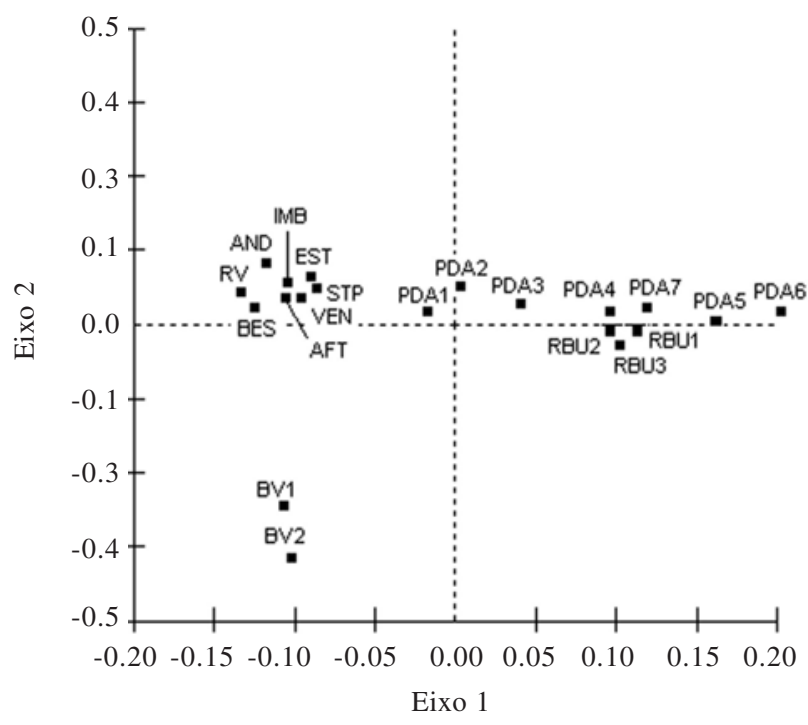

Figura 3. Relações florísticas entre os 20 fragmentos de Floresta Atlântica ombrófila de baixas altitudes na bacia do rio São João, RJ, Brasil, através da análise de coordenadas principais (método de distâncias médias). O eixo 1 explica $14,5 \%$ e o eixo 2 explica $13 \%$. Siglas dos fragmentos conforme Tab. 1. 
936 Carvalho, Nascimento \& Oliveira Filho: Composição, riqueza e heterogeneidade da flora arbórea da bacia do rio...

floras em diferentes escalas geográficas (Torres et al. 1997; Oliveira Filho \& Fontes 2000; Meira Neto \& Martins 2002). A composição de espécies arbóreas da bacia do rio São João mostrou relação com regiões distintas da Floresta Atlântica brasileira, baseado na análise das espécies indicadoras de Oliveira Filho \& Fontes (2000). Das 142 espécies apontadas por estes autores como indicadoras da Floresta Atlântica ombrófila de baixas altitudes da costa brasileira (PR, SP, RJ, ES e BA), 62 $(43,7 \%)$ foram registradas na bacia do rio São João (Tab. 3). Algumas espécies indicadoras de outras formações florestais, ausentes (Floresta Atlântica semidecídua de baixas e elevadas altitudes) ou presentes com baixa cobertura geográfica (Floresta Atlântica ombrófila de elevadas altitudes) na bacia do rio São João, também foram registradas, além de espécies de ampla ocorrência ('supertramps'). Das 51 espécies apontadas como de ampla ocorrência por Oliveira Filho \& Fontes (2000), 35 $(68,6 \%)$ estão presentes na bacia do rio São João, e, dentre estas, Alchornea triplinervia, Cabralea canjerana, Casearia sylvestris, Guapira opposita, Guarea guidonia, Mabea fistulifera, Nectandra oppositifolia e Tapirira guianensis estão também entre as de maior freqüência na Floresta Atlântica da bacia do rio São João (Tab. 4).

Tabela 3. Espécies apontadas por Oliveira Filho \& Fontes (2000) como indicadoras para as diversas fisionomias no domínio da Floresta Atlântica do Sudeste brasileiro, presentes nos fragmentos de Floresta Atlântica ombrófila de baixas altitudes da bacia do rio São João, RJ, Brasil. $\mathrm{n}=$ número de espécies; *espécies de maior freqüência nos fragmentos.

\begin{tabular}{|c|c|c|}
\hline Baixas altitudes & O. puberula & Picramnia glazioviana \\
\hline Ombrófila $(\mathrm{n}=62)$ & O. velutina & Platypodium elegans \\
\hline Aniba firmula & Parinari excelsa & Pouteria gardneri \\
\hline Astrocaryum aculeatissimum* & Pouteria caimito & Protium spruceanum \\
\hline Bathysa meridionalis & Plathymiscium floribundum & Siparuna guianensis \\
\hline Brosimum glaziovii & Pouroma guianensis & Tabebuia chrysotricha \\
\hline B. guianense* & Pseudobombax grandiflorum & Vismia guianensis \\
\hline Calyptranthes grandifolia & Pseudopiptadenia contorta* & \\
\hline Campomanesia guaviroba & Pterocarpus rohrii & Baixas e elevadas altitudes \\
\hline Carpotroche brasiliensis & Quiina glaziovii & Supertramps $(\mathrm{n}=35)$ \\
\hline Cedrela odorata & Simarouba amara & Alchornea glandulosa \\
\hline Chrysophyllum flexuosum & Sloanea guianensis & A. triplinervia* \\
\hline Coussapoa microcarpa & Solanum swartzianum & Andira fraxinifolia \\
\hline Cryptocarya moschata & Sparattosperma leucanthum* & Aspidosperma parvifolium \\
\hline Cupania emarginata & Swartzia flaemengii & Cabralea canjerana \\
\hline C. racemosa* & Tabebuia heptaphylla* & Calophyllum brasiliense \\
\hline Dalbergia nigra & Tachigali denudata & Cariniana estrellensis \\
\hline Ecclinusa ramiflora* & Trichilia elegans & Casearia sylvestris* \\
\hline Eriotheca pentaphylla & T. lepidota & Cecropia pachystachya \\
\hline Eugenia brasiliensis & Virola gardneri & Copaifera langsdorfii \\
\hline E. moroviana & & Cordia sellowiana \\
\hline Euterpe edulis* & Baixas altitudes & Croton floribundus \\
\hline Geissospermum laeve & Estacional $(\mathrm{n}=11)$ & Endlicheria paniculata \\
\hline Gomidesia spectabilis & Acacia polyphylla & Erythroxylum citrifolium \\
\hline Guatteria australis & Apuleia leiocarpa* & Guapira opposita* \\
\hline Himatanthus lancifolius & Chrysophyllum gonocarpum & Guarea guidonia* \\
\hline Inga capitata & Cupania oblongifolia* & G. macrophylla \\
\hline I. Vera & Ficus adhatifolia & Jacaratia spinosa \\
\hline I. striata & Guarea kunthiana & Luehea divaricata \\
\hline Jacaranda puberula & Siparuna guianensis & Mabea fistulifera* \\
\hline Jacaratia heptaphylla & Swartzia apetala & Maclura tinctoria \\
\hline Joannesia princeps & Tabernaemontana hystrix & Matayba guianensis \\
\hline Lecythis pisonis & Trichilia casaretti & Maytenus communis \\
\hline Licaria armeniaca & Xylopia sericea* & Myrcia rostrata \\
\hline Magnolia ovata & & Myrsine umbellata \\
\hline Marlierea suaveolens & Elevadas altitudes & Nectandra oppositifolia* \\
\hline Melanoxylon brauna & Ombrófila e estacional $(n=14)$ & Pera glabrata \\
\hline Micropholis crassipedicellata & Cecropia glaziovii & Piptadenia gonoacantha \\
\hline Mollinedia schottiana & Clethra scabra & Protium heptaphyllum \\
\hline Myrocarpus frondosus & Cryptocarya saligna & Sapium glandulatum \\
\hline Nectandra membranacea & Guatteria australis & Tabebuia serratifolia \\
\hline Ocotea dispersa & Miconia cinnamomifolia* & Tapirira guianensis* \\
\hline O. divaricata & Nectandra puberula & Trema micrantha \\
\hline O. elegans & Ocotea silvestris & Zanthoxylum rhoifolium \\
\hline
\end{tabular}


Tabela 4. Espécies arbóreas de maior freqüência $(\geq 50 \%)$ nos fragmentos de Floresta Atlântica ombrófila de baixas altitudes da bacia do rio São João, RJ, Brasil, ordenadas pelos valores decrescentes de freqüência relativa (FR). CS = classificação sucessional (sensu Gandolfi et al. 1995): $\mathrm{Pi}=$ pioneira; $\mathrm{Si}=$ secundária inicial; $\mathrm{St}=$ secundária tardia; $\mathrm{SD}=$ síndrome de dispersão (sensu Van der Pijl 1982): Zooc. $=$ zoocórica; Anemoc. $=$ anemocórica; Autoc. $=$ autocórica. $*$ Espécies que também apresentaram elevadas densidades nos fragmentos.

\begin{tabular}{|c|c|c|c|c|}
\hline Família & Espécie & CS & $\mathrm{SD}$ & $\mathrm{FR}(\%)$ \\
\hline ANNONACEAE & Xylopia sericea A. St.-Hil.* & $\mathrm{Pi}$ & Zooc. & 80,0 \\
\hline MELIACEAE & Cabralea canjerana (Vell.) Mart. & $\mathrm{Pi}$ & Zooc. & 80,0 \\
\hline MORACEAE & Brosimum guianense (Aubl.) Huber & $\mathrm{Si}$ & Zooc. & 80,0 \\
\hline NYCTAGINACEAE & Guapira opposita (Vell.) Reitz* & $\mathrm{Si}$ & Zooc. & 80,0 \\
\hline ARECACEAE & Astrocaryum aculeatissimum (Schott) Burret* & $\mathrm{Pi}$ & Zooc. & 75,0 \\
\hline FLACOURTIACEAE & Casearia sylvestris $\mathrm{SW} . *$ & $\mathrm{Si}$ & Zooc. & 75,0 \\
\hline FLACOURTIACEAE & Casearia arborea (Rich.) Urb. & $\mathrm{Si}$ & Zooc. & 70,0 \\
\hline LEGUMINOSAE & Pseudopiptadenia contorta (DC.) G.P. Lewis \& M.P. Lima* & $\mathrm{Pi}$ & Anemoc. & 70,0 \\
\hline ANACARDIACEAE & Tapirira guianensis Aubl.* & $\mathrm{Si}$ & Zooc. & 65,0 \\
\hline LACISTEMACEAE & Lacistema pubescens Mart.* & St & Zooc. & 65,0 \\
\hline LEGUMINOSAE & Apuleia leiocarpa (Vogel) J.F. Macbr. & $\mathrm{Si}$ & Anemoc. & 65,0 \\
\hline MELASTOMATACEAE & Miconia cinnamomifolia (DC.) Naudin* & $\mathrm{Pi}$ & Zooc. & 65,0 \\
\hline MORACEAE & Helicostylis tomentosa (Poepp. \& Endl.) Macbride & $\mathrm{Si}$ & Zooc. & 65,0 \\
\hline LAURACEAE & Nectandra oppositifolia Nees.* & $\mathrm{Si}$ & Zooc. & 60,0 \\
\hline MELIACEAE & Guarea guidonia (L.) Sleumer* & $\mathrm{Si}$ & Zooc. & 60,0 \\
\hline RUBIACEAE & Bathysa mendoncaei K. Schum. & St & Autoc. & 60,0 \\
\hline SAPOTACEAE & Ecclinusa ramiflora Mart. & St & Zooc. & 60,0 \\
\hline BIGNONIACEAE & Sparattosperma leucanthum Schum.* & $\mathrm{Pi}$ & Anemoc. & 55,0 \\
\hline EUPHORBIACEAE & Alchornea triplinervia (Spreng.) Müll. Arg. & $\mathrm{Si}$ & Zooc. & 55,0 \\
\hline EUPHORBIACEAE & Hieronyma alchorneoides Allemão & $\mathrm{Si}$ & Zooc. & 55,0 \\
\hline LEGUMINOSAE & Chamaecrista ensiformis (Vell.) H.S. Irwin \& Barneby & $\mathrm{Si}$ & Autoc. & 55,0 \\
\hline LEGUMINOSAE & Plathymenia foliolosa Benth. & St & Anemoc. & 55,0 \\
\hline MYRTACEAE & Myrcia fallax (Rich.) DC. & $\mathrm{Si}$ & Zooc. & 55,0 \\
\hline SAPINDACEAE & Cupania racemosa (Vell.) Radlk. & $\mathrm{Si}$ & Zooc. & 55,0 \\
\hline ARECACEAE & Euterpe edulis Mart.* & St & Zooc. & 50,0 \\
\hline ASTERACEAE & Vernonia discolor (Less.) H.Rob. & $\mathrm{Pi}$ & Anemoc. & 50,0 \\
\hline BIGNONIACEAE & Tabebuia heptaphylla (Vell.) Toledo & St & Anemoc. & 50,0 \\
\hline EUPHORBIACEAE & Mabea fistulifera Mart. & $\mathrm{Pi}$ & Zooc. & 50,0 \\
\hline RUBIACEAE & Psychotria velloziana Benth. & $\mathrm{Si}$ & Zooc. & 50,0 \\
\hline SAPINDACEAE & Cupania oblongifolia Mart.* & $\mathrm{Si}$ & Zooc. & 50,0 \\
\hline
\end{tabular}

Tais generalizações, apesar de tentarem fornecer uma visão mais apurada da vegetação, devem ser vistas com cautela, pois espécies típicas de determinadas formações florestais podem coexistir com outras mais freqüentes em outros tipos de formação, e determinadas espécies podem ser mais freqüentes e abundantes em uma formação e raras ou ausentes em outras. Com base nestas informações, sugere-se que, apesar da vegetação analisada na bacia do rio São João estar inserida no domínio da Floresta Ombrófila Densa (sensu Veloso et al. 1991), a proximidade com outras formações florestais, como as florestas estacionais de baixas altitudes do Norte Fluminense e regiões costeiras, e as florestas ombrófilas de elevadas altitudes na cadeia da Serra do Mar, parece exercer influência sobre sua composição de espécies arbóreas. Ressalta-se ainda que a fragmentação florestal nessa região resultou em manchas de vegetação muito heterogêneas quanto à composição arbórea, o que contribui para que algumas espécies estejam presentes em certas localidades e ausentes ou raras em outras, ao passo que aquelas mais adaptadas a distúrbios e com estratégias de dispersão do tipo colonizador possam ter expandido sua distribuição.

A relevância de estudos dessa natureza, como subsídio a estratégias de conservação, está muito mais em apontar onde e com que frequiência as espécies estão ocorrendo do que em definir limites para as diferentes formações florestais. Com este propósito, a Tab. 4 apresenta a lista das espécies arbóreas mais freqüentes nas florestas da bacia do rio São João, definidas pela sua ocorrência em pelo menos metade (50\%) dos 20 trechos florestais utilizados na análise. Algumas destas espécies também apresentaram elevada densidade relativa nos trechos analisados, tais como Astrocaryum aculeatissimum, Casearia sylvestris, Cupania oblongifolia, Euterpe edulis, Guapira opposita, Guarea guidonia, Lacistema pubescens, Miconia cinnamomifolia, Nectandra oppositifolia, Pseudopiptadenia contorta, Sparattosperma leucanthum, Tapirira guianensis e Xylopia sericea (Borém \& Ramos 2001; Borém \& 
Oliveira Filho 2002; Carvalho et al. 2006a; Guedes-Bruni et al. 2006a; b; Pessoa \& Oliveira 2006; Carvalho et al. 2007; G.M.S. Neves, dados não publicados; P.J.F.P. Rodrigues, dados não publicados). A maioria delas pertence ao grupo ecológico das secundárias iniciais (sensu Gandolfi et al. 1995), o que pode ser entendido como um reflexo da perturbação antrópica nos fragmentos florestais da região (Hubbell et al. 1999). De fato, a maior parte das florestas analisadas apresenta fortes indícios de alterações nos seus padrões estruturais, florísticos e sucessionais, em conseqüência da fragmentação florestal e distúrbios antrópicos intrínsecos a este processo (Carvalho et al. 2006a; Pessoa \& Oliveira 2006; Carvalho et al. 2007). Apesar disso, a maioria das espécies possui síndrome de dispersão biótica (zoocoria), sendo os diásporos dispersos principalmente pela avifauna (J.M. Correia, dados não publicados), o que significa que essas espécies podem ser utilizadas em programas de enriquecimento e recuperação de florestas perturbadas, ou para composição de corredores florestais conectando os fragmentos da bacia do rio São João (Reis \& Kageyama 2003; Silva 2003). Neste contexto, as espécies das famílias Lauraceae, Myrtaceae e Sapotaceae merecem um destaque especial, devido à sua conhecida apreciação por grandes mamíferos frugívoros, principalmente primatas (Tabarelli \& Peres 2002).

Vale salientar que na vegetação da bacia do rio São João estão presentes algumas espécies incluídas na Lista de Espécies da Flora Brasileira Ameaçadas de Extinção (IBAMA 1992): Brosimum glaziovii (rara) (Poço das Antas e União), Dalbergia nigra (vulnerável) (Boqueirão, Rio Vermelho e Imbaú), Melanoxylon brauna (vulnerável) (todas as localidades) e Mollinedia glabra (em perigo) (União); além de outras como Plathymenia foliolosa, citada por H.C. Lima (dados não publicados) como Leguminosa de relevante interesse conservacionista, devido à redução drástica de suas populações nas florestas do estado e ocorrência restrita a áreas com elevadas taxas de redução da cobertura florestal. Tais observações acentuam a importância das florestas da bacia do rio São João no cenário da conservação da flora arbórea da Floresta Atlântica, tanto em nível regional quanto nacional.

A presente análise apontou a flora arbórea da bacia do rio São João como detentora de alta diversidade em nível regional, podendo ser considerada como uma das mais ricas entre as de Floresta Atlântica ombrófila de baixas altitudes do sudeste brasileiro. Conforme a hipótese levantada, essa elevada diversidade deve-se à pronunciada heterogeneidade florística entre seus remanescentes florestais, e entre os fatores que mais influenciam nesta heterogeneidade, pode ser apontada a variação no estado de conservação dos remanescentes. Considerando que seus remanescentes estão inseridos em uma área de relevante interesse conservacionista, o Corredor Ecológico da Serra do Mar, mantendo inclusive diversas espécies arbóreas raras ou vulneráveis à extinção, medidas concretas visando a aumentar o número de unidades de conservação e a proteção efetiva das unidades de conservação já existentes na bacia do rio São João devem ser implementadas. Com a fragmentação florestal pretérita nesta região, restaram muitos fragmentos florestais de tamanhos reduzidos e localizados, em sua maioria, em propriedades privadas. A falta de opções de tamanhos e formatos desejáveis para implementação de novas unidades de conservação os torna praticamente as únicas alternativas para a preservação da biodiversidade remanescente. Portanto, as estratégias de conservação devem ter como alvo estas florestas particulares, incentivando o engajamento próativo dos proprietários na alocação de mais áreas como fragmentos destinados à conservação. Esse engajamento só será alcançado de forma realista quando o poder público recompensar de alguma forma a conservação por parte dos proprietários, ou então, indicar com clareza as vantagens diretas e indiretas da conservação sobre o patrimônio e bem estar dos proprietários.

\section{Agradecimentos}

Agradecemos a Fabiano Godoy e Márcio Schmidt, do Laboratório de geoprocessamento da Associação Mico Leão Dourado, pela confecção do mapa; ao Rodrigo Bacellar do IBAMA/Poço das Antas e responsável pela APA da Bacia do rio São João/Mico-Leão-Dourado, pela disponibilização de parte do material bibliográfico utilizado nas análises; ao FNMA/MMA, pelo apoio financeiro e ao Laboratório de Ciências Ambientais da Universidade Estadual do Norte Fluminense, Associação Mico Leão Dourado e IBAMA, pelo apoio logístico durante a coleta de parte dos dados; a CAPES, pela concessão da bolsa de mestrado ao primeiro autor no período 2003-2005.

\section{Referências bibliográficas}

Ayres, J.M.; Fonseca, G.A.B.; Rylands, A.B.; Queiroz, H.L.; Pinto, L.P.; Masterson, D. \& Cavalcanti, R.B. 2005. Os corredores ecológicos das florestas tropicais do Brasil. Sociedade Civil Mamirauá, Belém.

Borém, R.A.T. \& Ramos, D.P. 2001. Estrutura fitossociológica da comunidade arbórea de uma toposseqüência pouco alterada de uma área de Floresta Atlântica, no município de Silva Jardim-RJ. Revista Árvore 25: 131-140.

Borém, R.A.T. \& Oliveira-Filho, A.T. 2002. Fitossociologia do estrato arbóreo em uma toposseqüência alterada de Mata Atlântica, município de Silva Jardim-RJ, Brasil. Revista Árvore 26: $727-742$.

Budowisk, G. 1965. Distribution of tropical american rain forest species in the light of succesional processes. Turrialba 15: 40-42. 
Carvalho, F.A.; Nascimento, M.T.; Procópio de Oliveira, P.; Rambaldi, D.M. \& Fernandes, R.V. 2004. A importância dos remanescentes florestais da Mata Atlântica de baixada costeira fluminense para a conservação da biodiversidade na APA da Bacia do Rio São João/Mico-Leão-Dourado - RJ. Pp. 106-113. In: Anais do IV Congresso Brasileiro de Unidades de Conservação, v.1. Curitiba, Fundação $O$ Boticário de Proteção à Natureza: Rede Nacional Pró Unidades de Conservação.

Carvalho, F.A.; Nascimento, M.T. \& Braga, J.M.A. 2006a. Composição e riqueza florística do componente arbóreo da Floresta Atlântica submontana na região de Imbaú, município de Silva Jardim, RJ. Acta Botanica Brasilica 20: 727-740.

Carvalho, F.A.; Nascimento, M.T.; Braga, J.M.A \& Rodrigues, P.J.F.P. 2006b. Estrutura da comunidade arbórea da Floresta Atlântica de baixada periodicamente inundada na Reserva Biológica de Poço das Antas, Rio de Janeiro, Brasil. Rodriguésia 57: 503-518.

Carvalho, F.A.; Nascimento, M.T. \& Braga, J.M.A. 2007. Estrutura e composição florística do estrato arbóreo de um remanescente de Mata Atlântica submontana no município de Rio Bonito, RJ, Brasil (Mata Rio Vermelho). Revista Árvore 31: 717-730.

Cronquist, A. 1981. An integrated system of classification of flowering plants. New York, Columbia University Press.

Dean, W. 1996. A ferro e fogo - a história e a devastação da Mata Atlântica brasileira. São Paulo, Companhia das Letras.

Felfili, M.C. \& Felfili, J.M. 2001. Diversidade alfa e beta no cerrado sensu stricto da Chapada Pratinha, Brasil. Acta Botanica Brasilica 15: 243-254

Felfili, J.M.; Silva Junior, M.C.; Sevilha, A.C.; Fagg, C.W.; Walter, B.M.T.; Nogueira, P.E.N. \& Rezende, A.V. 2004. Diversity, floristic and structural patterns of cerrado vegetation in Central Brazil. Plant Ecology 175: 37-46.

Fundação SOS Mata Atlântica. 2002. Atlas da evolução dos remanescentes florestais e ecossistemas associados no domínio da Mata Atlântica no período 1995-2000. São Paulo, Fundação SOS Mata Atlântica/Instituto Nacional de Pesquisas Espaciais.

Gandolfi, S.; Leitão Filho, H.F. \& Bezerra, C.L.F. 1995 Levantamento florístico e caráter sucessional das espécies arbustivo-arbóreas de uma floresta mesófila semidecídua no município de Guarulhos, SP. Revista Brasileira de Biologia 55: $753-767$.

Gentry, A.H. 1988. Changes in plant community diversity and floristic composition on environmental and geographical gradients. Annals of Missouri Botanical Garden 75: 1-34.

Gentry, A.H. 1995. Patterns of diversity and floristic composition in neotropical montane forests. Pp. 103-126. In: S.P. Churchill; H. Baslev; E. Forero \& J.L. Luteyn (eds.). Biodiversity and Conservation of Neotropical Montane Forests: Proceedings of Neotropical Montane Forest Biodiversity and Conservation Symposium. New York, The New York Botanical Garden.

Guedes-Bruni, R.R.; Silva Neto, S.J.; Morim, M.P. \& Mantovani, W. 2006a. Composição florística e estrutura de trecho de Floresta Ombrófila Densa Atlântica aluvial na Reserva Biológica de Poço das Antas, Silva Jardim, Rio de Janeiro, Brasil. Rodriguésia 57: 413-428.

Guedes-Bruni, R.R.; Silva Neto, S.J.; Morim, M.P. \& Mantovani, W. 2006b. Composição florística e estrutura de dossel em trecho de Floresta Ombrófila Densa Atlântica sobre morrote mamelonar na Reserva Biológica de Poço das Antas, Rio de Janeiro, Brasil. Rodriguésia 57: 429-442.

Heltshe, J.F. \& Forrester, N.E. 1983. Estimating species richness using the jackknife procedure. Biometrics 39: 1-12

Hubbel, S.P.; Foster, R.B.; O’Brien, S.T.; Harms, K.E.; Condit, R.; Wechsler, B.; Wright, S.J. \& Lao, S.L. 1999. Light gaps disturbance, recruitment limitations and tree diversity in a Neotropical forest. Science 283: 554-557.

IBAMA. 1992. Lista de Espécies da Flora Ameaçadas de Extinção. Portaria $n^{\circ} 37-\mathrm{N}$ de 3 de abril de 1992. Brasília, Instituto Brasileiro do Meio Ambiente.
IBDF. 1981. Plano de manejo da Reserva Biológica de Poço das Antas. Documento Técnico $n^{\circ} 10$. Brasília, Instituto Brasileiro de Desenvolvimento Florestal.

Kent, M. \& Coker, P. 1992. Vegetation Description and analysis, a Practical Approach. London, Belhaven Press.

Kierulff, M.; Rambaldi, D.M. \& Kleiman, D.G. 2005. Passado, presente e futuro do mico-leão-dourado e de seu hábitat. Pp. 95-102. In: C. Galindo-Leal \& I.G. Câmara (eds.). Mata Atlântica: biodiversidade, ameaças e perspectivas. Belo Horizonte, Fundação SOS Mata Atlântica/Conservação Internacional.

Koeppen, W. 1948. Climatología. México, Editora Fondo de la Cultura Económica.

Kovach, W.L. 2004. MVSP (Multivariate Statistical Package), version $3.13 \mathrm{~m}$. Wales, Kovach Computing Service.

Kurtz, B.C. \& Araujo, D.S.D. 2000. Composição florística e estrutura do componente arbóreo de um trecho de Mata Atlântica na Estação Ecológica Estadual do Paraíso, Cachoeira de Macacú, Rio de Janeiro, Brasil. Rodriguésia 51: 69-112.

Lima, M.P.M. \& Guedes-Bruni, R.R. 1997. Reserva Ecológica de Macaé de Cima, Nova Friburgo - RJ: aspectos florísticos das espécies vasculares. Rio de Janeiro, Jardim Botânico do Rio de Janeiro.

Meira-Neto, J.A.A. \& Martins, F.R. 2002. Composição florística de uma floresta estacional semidecidual montana no município de Viçosa-MG. Revista Árvore 26: 437-446.

Moreno, M.R.; Nascimento, M.T. \& Kurtz, B.C. 2003. Estrutura e composição florística do estrato arbóreo em duas zonas altitudinais na Mata Atlântica de encosta da região do Imbé, RJ. Acta Botanica Brasilica 17: 371-386.

Mori, S.A.; Boom, B.M. \& Prance, G.T. 1981. Distribution patterns and conservation of eastern Brazilian coastal forest species. Brittonia 33: 233-245.

Oliveira Filho, A.T. \& Fontes, M.A.L. 2000. Patterns of floristic differentiation among Atlantic Forests in Southeastern Brazil and the influence of climate. Biotropica 32: 793-810.

Oliveira Filho; A.T.; Tameirão Neto; E.; Carvalho; W.A.C.; Werneck; M.; Brina; A.E.; Vidal; C.V.; Rezende; S.C. \& Pereira; J.A.A. 2005. Análise florística do compartimento arbóreo de áreas de Floresta Atlântica sensu lato na região das bacias do Leste (Bahia, Minas Gerais, Espírito Santo de Rio de Janeiro). Rodriguésia 55: $185-235$

Peixoto, G.L.; Martins, S.V.; Silva, A.F. \& Silva, E. 2004. Composição florística do componente arbóreo de um trecho de Floresta Atlântica na Área de Proteção Ambiental da Serra da Capoeira Grande, Rio de Janeiro, RJ, Brasil. Acta Botanica Brasilica 18: $151-160$.

Pereira, I.M.; Oliveira Filho, A.T.; Botelho, S.A.; Carvalho, W.A.C.; Fontes, M.A.L.; Schiavini, I. \& Silva, A.F. 2006. Composição florística do compartimento arbóreo de cinco remanescentes florestais do maciço do Itatiaia, Minas Gerais e Rio de Janeiro. Rodriguésia 57: 103-126.

Pessoa, S.V.A. \& Oliveira, R.R. 2006. Análise estrutural da vegetação arbórea em três fragmentos florestais na Reserva Biológica de Poço das Antas, Rio de Janeiro, Brasil. Rodriguésia 57: 391-412.

Primo, P.B. \& Volker, C.M. 2003. Bacias hidrográficas dos rios São João e das Ostras: águas, terras e conservação ambiental. Rio de Janeiro, Consórcio Intermunicipal Lagos São João.

Reis, A. \& Kageyama, P.Y. 2003. Restauração de áreas degradadas utilizando interações específicas. Pp. 91-110. In: P.Y. Kageyama; R.E. Oliveira; L.F.D. Moraes; V.L. Engel \& F.B. Gandara (orgs.). Restauração ecológica de ecossistemas naturais. Botucatu, FEPAP

Royal Botanical Garden. 1993. Index Kewensis on compact disc - manual. Oxford, Oxford University Press.

Scarano, F.R.; Ribeiro, K.T.; Moraes, L.F.D. \& Lima, H.C. 1997. Plant establishment on flooded and unflooded patches of a freshwater swamp forest in southeastern Brazil. Journal of Tropical Ecology 14: 793-803. 
940 Carvalho, Nascimento \& Oliveira Filho: Composição, riqueza e heterogeneidade da flora arbórea da bacia do rio...

Scarano, F.R.; Rios, R.I. \& Esteves, F.A. 1998. Tree species richness, diversity and flooding regime: case studies of recuperation after anthropic impact in brazilian flood-prone forests. International Journal of Ecological and Environmental Sciences 24: 223-235

Scarano, F.R. 2006. Plant community structure and function in a swamp forest within the Atlantic Rain Forest complex: a synthesis. Rodriguésia 57: 491-502.

Scudeller, V.V.; Martins, F.R. \& Sheperd, G.J. 2001. Distribution and abundance of arboreal species in the Atlantic ombrophilous dense forest in Southeastern Brazil. Plant Ecology 152 185-199.

Silva, W.R. 2003. A importância das interações planta-animal nos processos de restauração. Pp. 77-90. In: P.Y. Kageyama; R.E Oliveira; L.F.D. Moraes; V.L. Engel \& F.B. Gandara (orgs.). Restauração ecológica de ecossistemas naturais. Botucatu, FEPAP.
Tabarelli, M. \& Mantovani, W. 1999. A riqueza de espécies arbóreas na Floresta Atlântica de encosta no Estado de São Paulo (Brasil). Revista Brasileira de Botânica 22: 217-223.

Tabarelli, M. \& Peres, C.A. 2002. Abiotic and vertebrate seed dispersal in Brazilian Atlantic Forest: implications for forest regeneration. Biological Conservation 106: 165-176.

Thomaz, W.M.W.; Carvalho, A.M.V.; Amorim, A.M.A.; Garrison, J. \& Arbeláez, A.L. 1998. Plant endemism in two forests in southern Bahia, Brazil. Biodiversity and Conservation 7: 311-322.

Torres, R.B.; Martins, F.R. \& Kinoshita, L.S. 1997. Climate, soil and tree flora relationships in forest in the State of São Paulo, southeastern Brazil. Revista Brasileira de Botânica 20: 41-49.

Van der Pijl, L. 1982. Principles of dispersal in higher plants, $3^{\text {rd }}$. Berlin, Springer-Verlag.

Veloso, H.P.; Rangel Filho, A.L.R. \& Lima, J.C.A. 1991. Classificação da vegetação brasileira adaptada a um sistema universal. Rio de Janeiro, IBGE.

Versão eletrônica do artigo em www.scielo.br/abb e http://www.botanica.org.br/acta/ojs 